\title{
The initial stage of dam-break flow of two immiscible fluids. Linear analysis of global flow
}

\author{
Oguz Yilmaz ${ }^{\mathrm{a},{ }^{,},}$, Alexander Korobkin ${ }^{\mathrm{b}}$, Alessandro Iafratic \\ a Department of Mathematics, Izmir Institute of Technology, Urla, Izmir 35430, Turkey \\ ${ }^{\mathrm{b}}$ School of Mathematics, University of East Anglia, Norwich, UK \\ 'INSEAN-CNR, The Italian Ship Model Basin, Rome, Italy
}

\section{A R T I C L E I N F O}

\section{Article history:}

Received 11 March 2013

Received in revised form 9 April 2013

Accepted 17 April 2013

\section{Keywords:}

Immiscible fluids

Dam-break flow

Singular velocity

\begin{abstract}
A B S T R A C T
Two-dimensional impulsive flow of two immiscible fluids is studied within the potential flow theory. Initially the fluids of different depths and different densities are at rest and separated with a thin vertical plate. The plate is withdrawn suddenly and gravity-driven flow of the fluids starts. During the early stage the flow is described by the linear potential theory. Attention is paid to the motion of the interface between the fluids and the singular behaviour of the velocity field at the triple point, where the free surfaces of the fluids and the interface meet each other. The linear problem is solved by the Fourier series method. Local analysis of the flow field close to the triple point reveals that the singularity of the flow depends on the ratio of the fluid densities with a coefficient dependent on both the density ratio and the shape of the flow region. The flow velocity is also log-singular at the point where the interface meets the bottom. The intensity of this singularity depends on the density ratio. The latter singularity disappears when the densities of the fluids are equal. The Fourier series solution supplemented by the singularity analysis at the corner points resolves these initial singularities. Comparisons with solutions obtained through the boundary element method are established for validation purposes. The numerical analysis of the problem by the boundary element method is carried out and it compares quite well with the Fourier series solution. The singular flow field which leads to the jet formation at the initial instant has been observed by both methods. The problem of dam-break flow for the wet-bed case corresponds to the present problem with equal densities of the fluids. Comparisons with data available in literature are established in the case of fluids with the same density.
\end{abstract}

(c) 2013 Elsevier Ltd. All rights reserved.

\section{Introduction}

Plane unsteady problem of the gravity-driven flow of two immiscible fluids is considered. Initially the fluids are separated with a thin vertical plate and are at rest. The fluid depths are not equal to each other. At the initial time instant, $t^{\prime}=0$, the plate is instantly removed. The flow of two different fluids driven by the gravity starts suddenly (see Fig. 1). The present study is concerned with the initial stage of the flow, during which the fluid displacements are small compared with the fluid depths. The problem is studied in the Cartesian coordinate system $\left(x^{\prime}, y^{\prime}\right)$. A prime stands for dimensional variables. The line $y^{\prime}=$ 0 corresponds to the rigid bottom. The interval, $x^{\prime}=0,0<y^{\prime}<H^{-}$, corresponds to the initial position of the interface between the fluids. Quantities corresponding to the fluid which is originally on the left of the plate with the depth $H^{-}$are denoted by the superscript minus. Quantities describing the fluid on the right of the plate with the depth $\mathrm{H}^{+}$are denoted by the superscript plus. Densities of the fluids are $\rho^{-}$ and $\rho^{+}$, correspondingly. The ratio of the densities is denoted by $\gamma=$

\footnotetext{
* Corresponding author. Tel.: + 905323056511.

E-mail address: oguzyilmaz@iyte.edu.tr(O. Yilmaz).
}

$\rho^{-} / \rho^{+}$. In the present analysis, $H^{+}>H^{-}$and $0<\gamma<\infty$.

This problem was studied in the past for two identical fluids, $\gamma=$ 1 , and for $\gamma \neq 1$ but without the free surfaces of the fluids. The first problem is known as a wet-bed dam-break problem [1-4] and the second as a stratified mixing problem [5].

Both problems have been studied using the nonlinear shallow water equations, see Goater and Hogg [6] and Gill [7], p. 259, correspondingly. The shallow water approximation is based on the assumption that the vertical component of the flow velocity is negligible compared to the horizontal components and the pressure is hydrostatic. The stratified mixing in a dam-break problem was studied in [5] with the aim of extending the Large-Eddy Simulation (LES) method to small-scale mixing problems. A simplified configuration similar to that in Fig. 1 with $\mathrm{H}^{+}=\mathrm{H}^{-}$and rigid upper boundary was chosen to provide a test case with well defined initial and boundary conditions for a complex flow in a rectangular enclosed domain. Özgökmen et al. [5] wrote "When non-hydrostatic pressure is not modelled while nonlinearities are (i.e. in a hydrostatic model), the wave steepens unabated and may cause an artificial mixing event, depending on the turbulence closures employed. Also, the dispersion relations for internal waves differ in hydrostatic and non-hydrostatic 


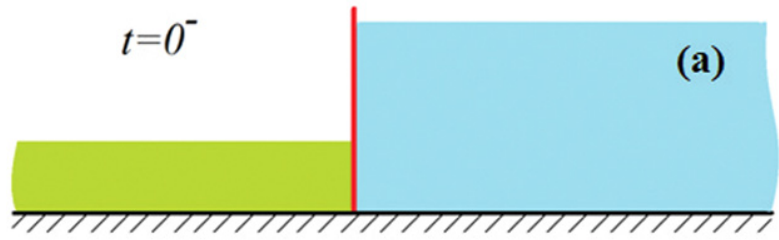

$t=0^{+}$

(b)

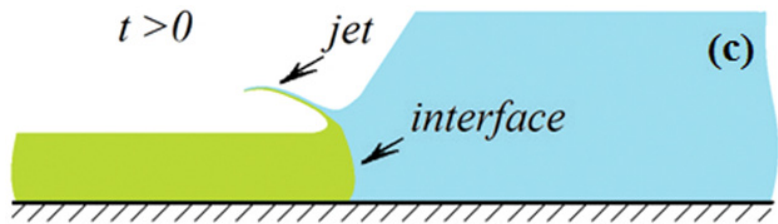

Fig. 1. Scheme of the gravity-driven flow: (a) initially the fluids are at rest and separated with a vertical plate; (b) the plate is removed and the flow starts at $t=0^{+}$; (c) expected flow of two immiscible fluids with the jet consisting both fluids.

cases. Thus, hydrostatic models not only are unable to correctly represent the shape of the internal waves and their degeneration into solitary waves, but they can also fail to represent the wave-induced boundary mixing."

The problem of wet-bed dam-break flow is very challenging. Even for the simplified geometry shown in Fig. 1, the initial conditions on the free surfaces of the fluids do not match each other which gives rise to a singularity of the initial flow at the triple point, where the free surfaces and the interface meet each other. This singularity and the resulting jet formed at the corner point shortly after the plate is withdrawn were discovered by Stansby et al. [1] for the fluids of equal densities. Both experimental and numerical studies of this new phenomenon were conducted in [1]. It was shown that the initial flow is singular at the corner point and a mushroom-like jet occurs just after release. The singularity was not studied. The jet observed in the experiments was well reproduced by the boundary-element method described by Cooker et al. [8]. To start the numerical simulations the singularity was smoothed out with the initial step-like free surface being approximated by a hyperbolic tangent. The obtained solutions were used by Lind et al. [4] (see Section 4.2.2.2 in [4]) to validate the method of incompressible smoothed particle hydrodynamics. The mushroom-like jets were also observed in experiments by Janosi et al. [2]. Gomez-Gesteira et al. [3] used these experimental results to demonstrate the capabilities of the smoothed particle hydrodynamics (SPH) method to reproduce the observed shapes of the free surface after the lock release. It is seen that the solution of the dam-break problem for wet bed case was used to validate some numerical algorithms.

The present analysis combines the stratified mixing problem and the dam-break problem for wet-bed case with focus on the local flow close to the triple point. The jet formed at this point is of interest in hydrodynamics of high-speed ships, where separation of the flow occurs at the intersection of the boards with the stern plane [9]. MartinezLegazpi [9] wrote "The difference in height between the separated stream and the free surface level immediately downstream the stern induces a transversal velocity component to the separated water mass that deflects its otherwise stream-like velocity towards the centerline of the hull. As a result, two symmetrical waves are formed that collide near the center plane of the wake." Taking advantage of the slender nature of the flow, the three-dimensional steady flow was approximated by a two-dimensional unsteady one. Then the flow close to the stern corresponds to the initial flow in a dam-break problem. Note that the latter problem differs from that studied by Stansby et al. [1] due to different initial conditions of the flow. The corner jets and resulting waves were studied experimentally, numerically and theoretically in [9].

Flow singularities at the intersection points between a liquid free surface and a solid boundary were studied intensively in the past. Initial stage of impulsive motion of a body piercing the free surface was analysed in $[10,11]$ for vertical and horizontal impacts of a semisubmerged circular cylinder, in $[12,13]$ for floating wedge and plate of zero draft, in [14-16] for horizontal impact of a vertical wall. It was shown that jet is developed at the intersection point with the jet strength being dependent on the angle between the body surface and the free surface of the liquid and the direction of the body motion. The flow in the jet region is nonlinear and self-similar during the early stage. Little is known about free-surface flows starting suddenly but with zero velocity. Initial flows generated by an accelerating plate were studied in [15] for vertical plate and in [16] for inclined plate. The papers $[15,16]$ are relevant to the present study, where the flow also starts from rest with zero initial velocity. It is interesting to note that the flow caused by uniformly accelerated vertical plate in [15] and the dam-break flow studied in [17] are locally similar close to the corresponding intersection points. In the present paper, the corresponding intersection point is at the bottom, where the interface between two fluids meets the solid boundary. However, there is another point, the triple point, which makes the problem more complicated than the problems mentioned above.

We assume a jet formed at the triple point. The shape of the jet and its structure can be different from that shown in Fig. 1c. One may expect a mushroom-like jet similar to that observed in [1] for fluids of equal densities. We expect that locally the jet flow is self-similar for small times after the flow starts. The structure of the jet should be investigated. In particular, we need to know portions of the fluids in the jet. This can explain the gravity-driven mixing of fluids. We expect that the local flow of immiscible fluids near the triple point is governed by the global flow. However the global flow depends weakly on the local one and can be approximately determined on its own during the early stage. Next, local flow can be recovered in properly stretched local coordinates and matched to the global flow. In this paper, we are concerned with the leading order global flow and matching conditions for the local region near the triple point. A formal small parameter is introduced to specify that only the initial stage of the flow is considered. The leading order global flow is described by a linear boundary value problem, which is obtained by linearisation of the boundary conditions and imposing them on the initial undisturbed positions of the boundaries of the fluids. The linear problem is solved by the Fourier series method and by the method of boundary element. The obtained velocity field is singular at both the triple point and intersection point between the interface and the flat bottom. The singularity of the flow is recovered with the help of local analysis and the Fourier method. The local analysis of the global flow at the triple point provides the order of the singularity and the solution obtained by the Fourier method provides the coefficient of the singularity. The boundary element method is used to verify the solution by the Fourier series method for different ratios of the fluid densities and different depths of the fluids.

The formulation of the small-time linearised problem is given in Section 2. In terms of uniformly valid small-time asymptotic solution of the fully nonlinear problem, in this paper, we restrict ourselves to the leading order outer solution. The numerical solution of this leading-order problem is obtained in Section 3 by the Fourier method. Local behaviour of the solution close to the triple point is studied in Section 4 . The coefficient of the singularity is determined by using the Fourier series solution. The boundary element method is applied 
to the problem in Section 5. The numerical solutions by the Fourier series method and the method of boundary element are compared in Section 6. Conclusions are drawn and future work is discussed in Section 7.

\section{Formulation of the problem}

The fluids are assumed ideal and incompressible. Surface tension and the presence of air are not included. The dam at $x^{\prime}=0$ disappears instantly at $t^{\prime}=0$. The resulting flow is assumed irrotational and twodimensional. We shall determine the liquid flow and the shape of its free surface during the early stage of the process.

The flow is described by the velocity potentials $\varphi^{\prime \pm}\left(x^{\prime}, y^{\prime}, t^{\prime}\right)$. The fluids are at rest when $t^{\prime}<0, \varphi^{\prime \pm}\left(x^{\prime}, y^{\prime}, 0^{-}\right)=0$. Initially the pressure distributions in the fluids are hydrostatic, $p^{\prime+}\left(x^{\prime}, y^{\prime}, 0^{-}\right)=\rho^{+} g\left(H^{+}-\right.$ $\left.y^{\prime}\right)$, where $x^{\prime}>0$ and $0<y^{\prime}<H^{+}$, and $p^{\prime-}\left(x^{\prime}, y^{\prime}, 0^{-}\right)=\rho^{-} g\left(H^{-}-y^{\prime}\right)$, where $x^{\prime}<0$ and $0<y^{\prime}<H^{-}$. Here $\rho^{ \pm}$are the corresponding fluid densities and $g$ is the gravity acceleration. The atmospheric pressure is taken as the reference pressure. At the initial time instant, $t^{\prime}=0$, the wall $x^{\prime}=0,0<y^{\prime}<H^{+}$is instantly removed and the gravity-driven flow starts. The part of the liquid boundary, where $x^{\prime}=0$ and $H^{-}<y^{\prime}$ $<H^{+}$, suddenly becomes free with $p^{\prime+}\left(x^{\prime}, y^{\prime}, 0^{+}\right)=0$ along it. This sudden change of the pressure must be balanced by the time derivative $-\rho^{+}\left(\partial \varphi^{\prime+} / \partial t^{\prime}\right)\left(0, y^{\prime}, 0^{+}\right)+p^{\prime}\left(0, y^{\prime}, 0^{-}\right)=0$ which follows from the unsteady Bernoulli equation. Note that the initial velocity of the flow is zero and the quadratic term in the Bernoulli equation gives a negligible contribution for small times. Correspondingly, along $x^{\prime}=$ 0 and $H^{-}<y^{\prime}<H^{+}$, the pressures $p^{\prime+}\left(0, y^{\prime}, 0^{-}\right)$and $p^{\prime-}\left(0, y^{\prime}, 0^{-}\right)$ are not equal to each other due to either different depths of the fluids or their different densities. However at $t^{\prime}=0^{+}$the pressures in the fluids must balance each other along the interface between the fluids. Again, it can be only achieved through the time derivatives of the velocity potentials. This observation indicates that the initial asymptotic solution can be sought in the form

$\varphi^{\prime \pm}\left(x^{\prime}, y^{\prime}, t^{\prime}\right) \sim t^{\prime} \phi^{\prime \pm}\left(x^{\prime}, y^{\prime}\right)$

during the early stage when the displacements of the fluid particles are much smaller than the fluid depths, $t^{\prime} \sqrt{g / H^{+}} \ll 1$.

It is convenient to introduce non-dimensional variables

$x^{\prime}=H^{+} x, \quad y^{\prime}=H^{+} y, \quad \phi^{\prime \pm}=g H^{+} \phi^{ \pm}, \quad p^{\prime \pm}=\rho^{ \pm} g H^{+} p^{ \pm}$.

In the non-dimensional variables the solution of the linearised problem depends on two parameters: density ratio $\gamma=\rho^{-} / \rho^{+}$and the depth ratio $\delta=H^{-} / H^{+}$. Linearization is a standard procedure as used in [17]; the velocity potentials, the free surfaces and the interface are expanded in a power series with the small non-dimensional parameter epsilon and as epsilon approaches zero we obtain the leading order solution. The boundary problems with respect to the new potentials $\phi^{ \pm}(x, y)$ are obtained by linearisation of the boundary conditions and imposing them on the initial undisturbed positions of the boundaries:

$\nabla^{2} \phi^{+}=0 \quad(x>0,0<y<1)$,

$\nabla^{2} \phi^{-}=0 \quad(x<0,0<y<\delta)$,

$\phi^{+}=0 \quad(x>0, y=1)$,

$\phi^{-}=0 \quad(x<0, y=\delta)$,

$\phi^{+}=1-y \quad(x=0, \delta<y<1)$,

$\phi^{+}+y-1=\gamma \phi^{-}+\gamma(y-\delta), \quad \phi_{x}^{+}=\phi_{x}^{-} \quad(x=0,0<y<\delta)$,

$\phi_{y}^{ \pm}=0 \quad(y=0)$,

$\phi^{ \pm} \rightarrow 0 \quad(x \rightarrow \pm \infty)$.

The boundary problem (2)-(9) is depicted in Fig. 2.

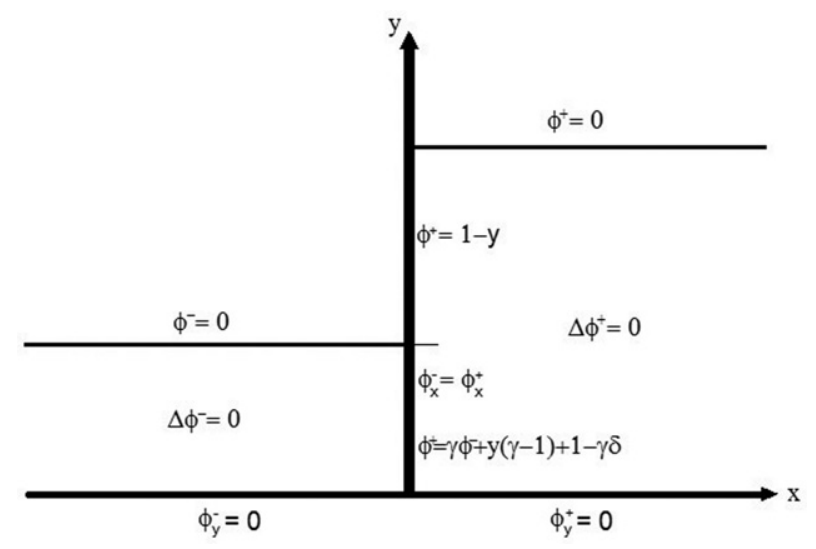

Fig. 2. Scheme of the linearised problem of two-fluid dam break.

The conditions (4) and (5) follow from the dynamic boundary conditions on the initially horizontal parts of the fluid boundaries; condition (6) states that the dynamic pressure balance the hydrostatic pressure on the initially vertical part of the fluid free surface. Conditions (7) on the interface between the two fluids imply that both the pressures in the fluids and the normal velocities of the flows are equal to each other on the interface. Condition (8) is applied on the bottom of the fluids, and condition (9) means that the fluids are at rest in the far field. The boundary problem describes the gravity-driven flow of two immiscible fluids during the early stage everywhere in the flow domain where the displacements and velocities of the fluid particles are finite. Note that the conditions (5) and (6) do not match each other at the corner point $x=0, y=\delta$. Also the conditions (7) and (8) do not match each other at the intersection point $x=0, y=$ 0 . At both points, singularities of the flow are expected within the model (2)-(9). The solution of this problem provides the leading order flow outside small vicinities of these two points during the early stage. The linear solution also provides matching conditions to formulate the inner problems in these vicinities, as well as sizes of the vicinities.

Once the problem (2)-(9) has been solved, the positions of the fluid boundaries in the dimensional variables are given by the following formulae

$y^{\prime} \approx H^{+}+\frac{1}{2} g t^{\prime 2} \phi_{y}^{+}\left(\frac{x^{\prime}}{H^{+}}, 1\right)\left(x^{\prime}>0\right), \quad y^{\prime} \approx H^{-}+\frac{1}{2} g t^{\prime 2} \phi_{y}^{-}\left(\frac{x^{\prime}}{H^{+}}, \delta\right)\left(x^{\prime}<0\right),(10)$

$x^{\prime} \approx \frac{1}{2} g t^{\prime 2} \phi_{x}^{+}\left(0, \frac{y^{\prime}}{H^{+}}\right) \quad\left(0<y^{\prime}<H^{+}\right)$

during the early stage. The shape of the interface between the two fluids is obtained from (11), where $0<y^{\prime}<H^{-}$.

The problem (2)-(9) is solved below numerically. There are several methods which can be applied to this problem. Analytic function theory combined with the Schwarz Christofel conformal mapping is one of them [9]. However, its application to our problem presents some difficulties due to the interface between the fluids. The method of eigenfunction expansions was used in [16]. This method being applied to our problem would require eigenfunction expansions constructed in local polar coordinates with the origins at the triple point, at the top corner point and at the bottom intersection point. The obtained expansions combined with the Fourier series solutions away from the three points could be truncated and the coefficients in the five expansions can be determined by the collocation method. In this paper more straight forward methods of Fourier series and boundary element are used. These methods are supported by the local analysis of the flows near the corner points, cross-validated and verified in terms of convergence. 


\section{Solution by the Fourier series method}

Separation of variables applied to both flow regions provides the following series for the velocity potential,

$\phi^{+}=\sum_{n=0}^{\infty} c_{n} e^{-\mu_{n} x} \cos \left(\mu_{n} y\right)$,

$\phi^{-}=\sum_{n=0}^{\infty} d_{n} e^{\mu_{n} x / \delta} \cos \left(\frac{\mu_{n} y}{\delta}\right)$

where $\mu_{n}=(2 n+1)(\pi / 2)$ and the coefficients $c_{n}$ and $d_{n}$ are to be determined. The potentials (12) and (13) satisfy the Laplace equation (2) and (3), the boundary conditions (4), (5), (8) and the condition at infinity (9). The conditions (6) and (7) serve to calculate the coefficients $c_{n}$ and $d_{n}$ in (12) and (13). The boundary conditions (7) at $x=$ 0 provide the following equations:

$\sum_{n=0}^{\infty} c_{n} \cos \left(\mu_{n} y\right)-1+y=\gamma \sum_{n=0}^{\infty} d_{n} \cos \left(\frac{\mu_{n} y}{\delta}\right)+\gamma(y-\delta), 0<y<\delta$,

$-\sum_{n=0}^{\infty} c_{n} \mu_{n} \cos \left(\mu_{n} y\right)=\sum_{n=0}^{\infty} \frac{\mu_{n}}{\delta} d_{n} \cos \left(\frac{\mu_{n} y}{\delta}\right), \quad 0<y<\delta$,

Multiplying (12) by $\cos \left(\mu_{m} y\right)$ and then integrating the resulting equation in $y$ from 0 to 1 , using the condition (6) and the first condition in (7), we obtain the following relation between the coefficients $c_{n}$ and $d_{n}$

$c_{n} / 2-\gamma \sum_{m=0}^{\infty} \alpha_{n m} d_{m}=f_{n}, \quad n=0,1, \cdots$,

where

$f_{n}=\frac{1}{\mu_{n}^{2}}\left[1-\gamma+\gamma \cos \left(\mu_{n} \delta\right)\right]$,

$\alpha_{n m}=-(-1)^{m} \frac{\mu_{m} \cos \left(\mu_{n} \delta\right) / \delta}{\mu_{n}^{2}-\mu_{m}^{2} / \delta^{2}}$.

Multiplying (15) by $\cos \left(\mu_{m} y / \delta\right)$ and then integrating the resulting equation in $y$ from 0 to $\delta$, we obtain another equation relating the coefficients $c_{n}$ and $d_{n}$,

$-\sum_{n=0}^{\infty} c_{n} \mu_{n} \alpha_{n m}=d_{m} \frac{\mu_{m}}{2}, \quad m=0,1, \ldots$

Now let us truncate the two infinite series in (12) and (13) to $N$ and $M$ terms respectively,

$\phi^{+}=\sum_{n=0}^{N} c_{n} e^{-\mu_{n} x} \cos \left(\mu_{n} y\right)$,

$\phi^{-}=\sum_{n=0}^{M} d_{n} e^{\mu_{n} x / \delta} \cos \left(\frac{\mu_{n} y}{\delta}\right)$.

Combining the truncated linear algebraic Eqs. (16) and (19) we get a linear system of $M+1$ equations for the coefficients $d_{\ell}$,

$\sum_{\ell=0}^{M}\left[\frac{\mu_{\ell}}{2} \delta_{\ell m}+2 \gamma \sum_{n=0}^{N} \mu_{n} \alpha_{n m} \alpha_{n l}\right] d_{\ell}=-2 g_{m}, \quad m=0,1,2, \ldots, M$,

where

$g_{m}=\sum_{n=0}^{N} \frac{\alpha_{n m}}{\mu_{n}}\left(1-\gamma+\gamma \cos \left(\mu_{n} \delta\right)\right)$,

and $\delta_{\ell m}=1$ when $\ell=m$ and $\delta_{\ell m}=0$ when $\ell \neq m$. The coefficients $c_{n}$ are obtained from (16) after solving (20) for $d_{\ell}$. The numbers $M$ and $N$ of terms in the truncated series must be big enough to ensure convergence of the numerical solution. Also, these numbers $M$ and $N$ should relate to each other. But, we still have to decide how many modes are needed to describe the flow in the two regions. It is suggested by Dalrymple [18] and Hudde and Letens [19] that $N>N_{S}=M / \delta$, where $\delta$ $<1$. This is necessary to ensure that similar modes in each region are matched. In fact, numerical tests suggest that $N=2 N_{S}$ in (20). After calculating the coefficients $c_{n}$ and $d_{n}$, boundary conditions (6) and (7) are checked numerically. It is observed that the equality of horizontal velocities at the interface is the most difficult one to satisfy. So, the number of modes, $M$, is determined by that condition.

\section{Local flows at the corner points}

In this section we identify the nature of the flow singularity at the corner points. It is convenient to rewrite the leading order problem (2)-(9) in terms complex velocities $\omega^{+}(z)$ and $\omega^{-}(z), z=x+i y$, defined in the semi strips $(x>0,0<y<1)$ and $(x<0,0<y<\delta)$ respectively. Here $\omega^{+}(z)=u^{+}-i v^{+}$and $\omega^{-}(z)=u^{-}-i v^{-}$are analytic in their own regions. The boundary conditions for $\omega^{+}(z)$ follow from (4), (6) and (8):

$u^{+}=0(y=1, x>0), \quad v^{+}=0(y=0, x>0), \quad v^{+}=-1(x=0, \delta<y<1)$,

and the conditions for $\omega^{-}(z)$ from (5) and (8):

$u^{-}=0(y=\delta, x>0), \quad v^{-}=0(y=0, x>0)$.

The conditions ( 7 ) at the interface $(x=0,0<y<\delta)$ have the form

$v^{+}+1=\gamma\left(v^{-}+1\right), \quad u^{+}=u^{-}$.

Local flow near the triple point $(0, \delta)$ is governed by the boundary conditions on the boundaries which meet each other at that point. This flow strongly depends on the density ratio $\gamma$. Three reference cases concerning $\gamma$ are considered:

1. The liquid on the left is much lighter than the liquid on the right. In this case, $\gamma \ll 1$ and the flow of the heavier liquid on the right can be determined in the leading order as $\gamma \rightarrow 0$ without accounting for the presence of the liquid on the left. The corresponding flow is that of dam break studied in [17]. The leading order flow of the lighter liquid is obtained by imposing the horizontal velocity, given by the solution of the dam break problem, at the interface. The latter flow is similar to that studied by King and Needham [15] for uniformly accelerating vertical plate but with the acceleration of the plate depending on the vertical coordinate. The local behaviour of this flow is discovered in [15] with log singularity of the vertical velocity of the flow at $(0, \delta)$. The correction to the leading order flow on the right caused by the presence of the lighter liquid is estimated as $O(\gamma)$ when $\gamma \rightarrow 0$ which follows from (21).

2. Both liquids are of the same density. In this case $\gamma=1$ and the complex velocities $\omega^{+}(z)$ and $\omega^{-}(z)$ are continuous at the interface which follows from (21). Then we can drop the superscripts "+" and "-" from the velocity terms, conditions at the interface (21) and consider a simple complex velocity $\omega(z)$. The resultant local boundary value problem for $\omega(z)$ is depicted in Fig. 3.

There are many (singular) solutions of this problem, we choose the least singular one

$u-i v=D_{1}(z-i \delta)^{-1 / 3}+i t$,

where $D_{1}$ is a constant which depends on the global solution. The solution (22) describes the local flow with finite kinetic energy.

3. The liquid on the left is much heavier than the liquid on the right. In this case $\gamma \gg 1$ and the flow of the left fluid is that of dam break. The leading order flow of the lighter liquid on the right is obtained by imposing the horizontal velocity given by the solution of the dam break problem at the interface, $x=0,0<y<\delta$. As for the case $\gamma \ll$ 1 , in the limit as $y \rightarrow 0^{+}$the solution is still similar to that studied by King and Needham [15]. However, differently from a classical dam break problem, boundary conditions of different type are applied on 


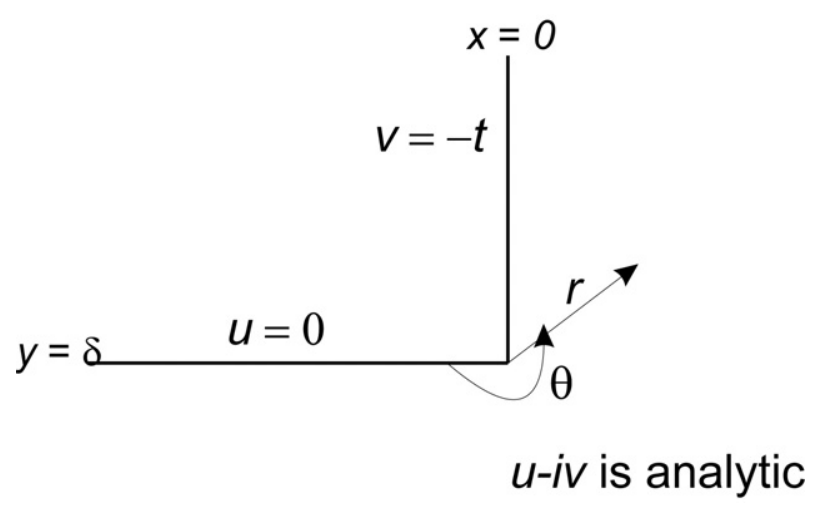

Fig. 3. Boundary value problem describing the local flow for $\gamma=1$.

the boundary $x=0$ for $y<\delta$ and for $y>\delta$. Due to the change in the condition at the triple point $x=0, y=\delta$, the problem is a mixed boundary value problem similar to the one studied in [13]. It is known that the solution of this problem describes the flow with square root singularity of the velocity at the point where the boundary condition changes its type [13].

The leading order complex velocity $\omega^{+}(z)$ when $\gamma \rightarrow \infty$ behaves at the point $(0, \delta)$ as

$\omega^{+}(z) \sim D_{\infty} e^{i \pi / 4}(z-i \delta)^{-1 / 2}+i t \quad(z \rightarrow i \delta)$,

where $D_{\infty}$ is a constant which depends on the global solution.

This analysis indicates that the singularity of the flow at the corner point depends on the parameter $\gamma$ and varies from 0 to $-1 / 2$ when $\gamma$ increases from zero to infinity. Assume that

$\omega^{+}(z) \sim D(z-i \delta)^{-\alpha(\gamma)}, \quad \omega^{-}(z) \sim E(z-i \delta)^{-\alpha(\gamma)}$

as $|z-i \delta| \rightarrow 0$, where the complex valued constants $D$ and $E$ depend on the global flow and $0 \leq \alpha \leq 1 / 2$. In the local polar coordinates $z-$ $i \delta=r \exp (i \theta)$, we have

$\omega^{+}(z) \sim D r^{-\alpha} \exp (-i \alpha \theta) \quad\left(\frac{-\pi}{2}<\theta<\frac{\pi}{2}, r \rightarrow 0\right)$,

$\omega^{-}(z) \sim E r^{-\alpha} \exp (-i \alpha \theta) \quad\left(-\pi<\theta<\frac{-\pi}{2}, r \rightarrow 0\right)$.

From the boundary conditions (5) and (6) we deduce that $E \exp (i \alpha \pi)$ is pure imaginary, say, $i E_{R}$ and that $D \exp (-i \alpha \pi / 2)$ is real, say, $D_{R}$. We conclude that the complex velocities near the corner point behave as

$$
\begin{aligned}
& \omega^{+}(z)=u^{+}-i v^{+} \sim D_{R} r^{-\alpha} \exp \left(i \alpha\left(\frac{\pi}{2}-\theta\right)\right) \quad\left(\frac{-\pi}{2}<\theta<\frac{\pi}{2}, r \rightarrow 0\right), \\
& \omega^{-}(z)=u^{-}-i v^{-} \sim i E_{R} r^{-\alpha} \exp (-i \alpha(\pi+\theta)) \quad\left(-\pi<\theta<\frac{-\pi}{2}, r \rightarrow 0\right) .
\end{aligned}
$$

The matching conditions (21) at the interface, $\theta=-\pi / 2$, provide relations between $\alpha$ and $\gamma$ and the coefficients $E_{R}$ and $D_{R}$,

$\alpha=\frac{2}{\pi} \arcsin \sqrt{\frac{\gamma}{2(\gamma+1)}}, \quad E_{R}=\frac{D_{R}}{\sqrt{\gamma(\gamma+1) / 2}}$.

It is possible to check by direct calculation that

$\alpha(0)=0, \quad \alpha(1)=\frac{1}{3}, \quad \alpha(\infty)=\frac{1}{2}$

which correspond to the results of our analysis of three reference cases. It is reasonable to assume that $D_{R}<0$. If so, the free surface near the corner point moves outwards the liquid region. When $\gamma \rightarrow$ 0 , Eq. (26) yield $\alpha=O(\sqrt{\gamma}), D_{R}=O(1)$ and $E_{R} \sim E_{R 0} / \sqrt{\gamma}$, where the real coefficients $D_{R}$ and $E_{R 0}$ are to be determined. Then formula (25) gives

$\omega^{-}(z) \sim i \frac{E_{R 0}}{\sqrt{\gamma}} e^{-\alpha \log r} \exp [-i \alpha(\pi+\theta)]$, where $r \rightarrow 0$ and $\gamma \rightarrow 0$. By using the expansion

$e^{-\alpha \log r}=1-\alpha \log r+O(\gamma)$

as $\gamma \rightarrow 0$ and $\alpha \log r \rightarrow 0$, formula (27) can be presented as

$\omega^{-}(z)-i \frac{E_{R 0}}{\sqrt{\gamma}} \sim-i E_{R 0} \frac{\sqrt{2}}{\pi} \log r$,

where the right hand side correctly describes the log singularity studied by King and Needham [15]. When $\gamma=1$, then $D_{R}=E_{R}, \alpha=1 / 3$ and the formulas in (25) coincide with (22) where $D_{1}=D_{R}$. When $\gamma$ $\rightarrow \infty, \omega^{-}(z)$ is not singular at the triple point in the leading order and $\omega^{+}(z)$ is square root singular. Indeed, Eq. (26) with $D_{R}=O(1)$ as $\gamma \rightarrow$ $\infty$ provide that $E_{R}=O(1 / \gamma)$ and there is no singularity in $\omega^{-}(z)$ in the leading order.

At the corner point, where the interface meets the bottom, $x=0$ and $y=0$, the complex velocities in the leading order as $r \rightarrow 0$ behave as

$\omega^{+}(z) \sim A \log z=A(\log r+i \theta) \quad\left(0 \leq \theta \leq \frac{\pi}{2}, r \rightarrow 0\right), z=r e^{i \theta}$,
$\omega^{-}(z) \sim B(\log z-i \pi)=B(\log r+i \theta-i \pi) \quad\left(\frac{\pi}{2} \leq \theta \leq \pi, r \rightarrow 0\right)$,

where $A$ and $B$ are real coefficients. The boundary conditions (8) at the bottom are satisfied with the asymptotic solutions (28). The form of these asymptotic formulas is inspired by the solution of the dambreak problem [17], which can be obtained from the present formulation when $\gamma=0$. Component wise, we find

$u^{+} \sim A \log r, \quad v^{+} \sim-A \theta, \quad u^{-} \sim B \log r, \quad v^{+} \sim B(\pi-\theta)$

near the corner point. At the interface between the two fluids, $\theta=$ $\pi / 2$ and the matching conditions (21) provide

$B=A, \quad A=\frac{2}{\pi} \frac{1-\gamma}{1+\gamma}$.

The formulas (29) predict that $A=2 / \pi$ when $\gamma=0$, which corresponds to the result by Korobkin and Yilmaz [17] (see Eq. (13)), and that there is no singularity of the flow if $\gamma=1$. The interface moves to the left if $\gamma<1$ and to the right if $\gamma>1$. Note that the leading order behaviour of the velocity field near the bottom point is known prior to the numerical analysis of the problem. The situation with the triple point $(0, \delta)$ is different. In (25), the power $\alpha$ of the singularity is given by (26) but the coefficient $E_{R}$ should be determined by using the solution of the global problem (2)-(9). To determine the coefficient $E_{R}$ in the local asymptotic formulae (25), we assume that the solution of the global problem (2)-(9) by the Fourier series method is known. Then the coefficients $d_{n}, n \geq 0$, are known as solutions of the system (20). Asymptotic behaviour of these coefficients as $n \rightarrow \infty$ is governed by the singularity of the solution at the triple point, which is the strongest singularity of the solution. The second equation in (25) makes it possible to decompose the horizontal velocity $u^{-}(0, y)$ at the interface, $0<y<\delta$, as

$u^{-}(0, y)=E_{R}(\delta-y)^{-\alpha} \sin \left(\frac{\alpha \pi}{2}\right)+\tilde{u}^{-}(0, y)$,

where $\tilde{u}^{-}(0, y)$ is log-singular at $y=0$ according to (28) and can also be singular at the triple point $y=\delta$ but this singularity is weaker than that of the first term in (30). Substituting (13) in (30), multiplying both sides by $\cos \left(\mu_{m} y / \delta\right)$ and integrating the resulting equation in $y$ from 0 to $\delta$, we obtain

$$
\frac{1}{2} d_{n} \mu_{n}=E_{R} \sin \left(\frac{\alpha \pi}{2}\right) \int_{0}^{\delta}(\delta-y)^{-\alpha} \cos \left(\frac{\mu_{n} y}{\delta}\right) d y+\int_{0}^{\delta} \tilde{u}^{-}(0, y) \cos \left(\frac{\mu_{n} y}{\delta}\right) d y .
$$

The second integral in (31) decays faster than the first one when $n \rightarrow \infty$. Therefore, the leading order asymptotic behaviour of the coefficients $d_{n}$ as $n \rightarrow \infty$ depends on asymptotic behaviour of the first integral in (31). By algebra we obtain the following asymptotic formula for this integral

$\int_{0}^{\delta}(\delta-y)^{-\alpha} \cos \left(\frac{\mu_{n} y}{\delta}\right) d y \sim\left(\frac{\delta}{\mu_{n}}\right)^{1-\alpha}\left\{\sin \left(\mu_{n}\right) \int_{0}^{\mu_{n}} \frac{\sin \lambda}{\lambda^{\alpha}} d \lambda+O\left(\frac{1}{n}\right)\right\} \quad(n \rightarrow \infty)$. 


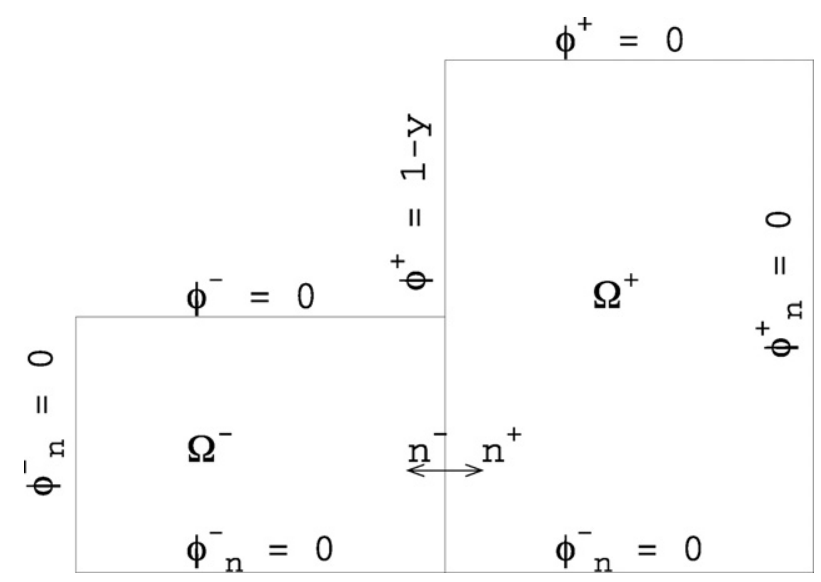

Fig. 4. Sketch of the domains and of the boundary conditions used for the solution of the leading order problem by the boundary element method.

Substituting the asymptotic forms of the integrals in (31) we have

$\frac{d_{n} \mu_{n}}{2} \sim E_{R}(-1)^{n} \sin \left(\frac{\alpha \pi}{2}\right)\left(\frac{\delta}{\mu_{n}}\right)^{1-\alpha} S(\gamma)$

for large $n$, where

$S(\gamma)=\int_{0}^{\infty} \frac{\sin \lambda}{\lambda^{\alpha}} d \lambda=\Gamma(1-\alpha) \sin \left(\frac{(1-\alpha) \pi}{2}\right)$

(see p. 439 in [20]). The formula (32) can be rewritten as

$\frac{(-1)^{n} \mu_{n}^{2-\alpha} d_{n}}{2 \delta^{1-\alpha} \sin (\alpha \pi / 2) S(\gamma)} \sim E_{R} \quad(n \rightarrow \infty)$,

where the left-hand side $E_{R}^{(n)}$ is known as a function of $n$ once the system (20) for the coefficients $d_{n}$ has been solved. The asymptotic formula (33) provides that the coefficient $E_{R}$ in (25) can be calculated as the limit of $E_{R}^{(n)}$ when $n \rightarrow \infty$, where $E_{R}^{(n)}$ are obtained by the Fourier series method.

\section{Solution of the leading order problem by boundary element approach}

The solution of the boundary value problem (2)-(9) governing the leading order velocity potentials is also derived by the boundary element approach. The boundary element method is used to derive an alternative solution of the problem (2)-(9) with the aim to verify the solution by the Fourier series method obtained in Section 3. The governing equations are basically the same as those discussed in Section 2 although, for convenience, a finite extension of the fluid domains is considered now. Hence, the problem is reduced to the solution of the two-dimensional Laplace equation in two finite domains together with the boundary conditions of the Neumann and Dirichlet type. As shown in Fig. 4, the left and right subdomains are closed by solid boundaries, positions of which are chosen far enough not to affect the solution substantially. In the simulations presented in Section 6 , the boundaries are located at $x=-20$ and $x=+20$ for the left and the right hand sides, respectively.

At the interface between the two sub-domains $\Omega^{ \pm}$both velocity potentials $\phi^{ \pm}(0, y)$ and their normal derivatives $\partial \phi^{ \pm} / \partial n^{ \pm}$are to be determined. Additional relations which couple the solutions in the two domains are given by conditions (7) rewritten here in a slightly different form for convenience:

$$
\begin{aligned}
& \frac{\partial \phi^{-}}{\partial n^{-}}=-\frac{\partial \phi^{+}}{\partial n^{+}}, \\
& \phi^{+}-\gamma \phi^{-}=\gamma(y-\delta)-(y-1) .
\end{aligned}
$$

In order to derive the solutions of the boundary value problems in the two domains, the corresponding velocity potentials are written in the form of a boundary integral representation. For the left subdomain $\Omega^{-}$we have

$\phi^{-}\left(\mathbf{x}_{P}\right)=\int_{\partial \Omega^{-}}\left(\frac{\partial \phi^{-}\left(\mathbf{x}_{Q}\right)}{\partial n_{Q}} G\left(\mathbf{x}_{P}-\mathbf{x}_{Q}\right)-\phi^{-}\left(\mathbf{x}_{Q}\right) \frac{\partial G\left(\mathbf{x}_{P}-\mathbf{x}_{Q}\right)}{\partial n_{Q}}\right) \mathrm{d} S_{Q}$,

which is valid for any point $\boldsymbol{x}_{P} \in \Omega^{-}$and $\boldsymbol{x}_{Q} \in \partial \Omega^{-}$. A similar representation holds for the velocity potential $\phi^{+}$inside the right subdomain $\Omega^{+}$. In Eq. (36) $G(\boldsymbol{x})$ is the free space Green's function for the Laplace operator in two dimensions.

Eq. (36) provides the velocity potential at any internal point, provided both the velocity potential and its normal derivative are known along the boundary. As only one of the two data, at most, is available on different portions of the boundary, in order to derive the other, Eq. (36) is written in the limit as $\boldsymbol{x}_{P} \rightarrow \partial \Omega^{-}$

$\frac{1}{2} \phi^{-}\left(\mathbf{x}_{P}\right)=\int_{\partial \Omega^{-}}\left(\frac{\partial \phi^{-}\left(\mathbf{x}_{Q}\right)}{\partial n_{Q}} G\left(\mathbf{x}_{P}-\mathbf{x}_{Q}\right)-\phi^{-}\left(\mathbf{x}_{Q}\right) \frac{\partial G\left(\mathbf{x}_{P}-\mathbf{x}_{Q}\right)}{\partial n_{Q}}\right) \mathrm{d} S_{Q}$

Solution of the above equation is derived numerically by discretizing the domain boundary into straight line segments with piecewise constant distributions of both the velocity potential and its normal derivative. The discretised form of Eq. (37) is

$$
\begin{aligned}
& a_{i} \phi_{i}^{-}-\sum_{j=1}^{N_{F}^{-}} \phi_{n, j}^{-} g_{i j}-\sum_{j=N_{F}^{-}+1}^{N_{F}^{-}+N_{I}} \phi_{n, j}^{-} g_{i j}+\sum_{j=N_{F}^{-}+1}^{N_{F}^{-}+N_{I}} \phi_{j}^{-} d_{i j}+\sum_{j=N_{F}^{-}+N_{I}+1}^{N_{F}^{-}+N_{I}+N_{N}^{-}} \phi_{j}^{-} d_{i j} \\
& =e_{i} \phi_{i}^{-}-\sum_{j=1}^{N_{F}^{-}} \phi_{j}^{-} d_{i j}+\sum_{j=N_{F}^{-}+N_{I}+1}^{N_{I}+N_{I}+N_{N}^{-}} \phi_{n, j}^{-} g_{i j},
\end{aligned}
$$

where $N_{F}^{-}$is the number of segments used to represent the free surface, $N_{I}$ is the number of segments at the interface and $N_{N}^{-}$is the number of segments adopted for the discretization of the bottom and far-field boundaries. In (38) $g_{i j}$ and $d_{i j}$ denote the influence coefficients of the segment $j$ on the midpoint of the segment $i$ related to $G$ and $\partial G / \partial n$, respectively. In the above equation the coefficients $\left(a_{i}, e_{i}\right)$ are $(1 / 2,0)$ if the velocity potential is unknown on panel $i$, whereas they take $(0,1 / 2)$ if the velocity potential is assigned at that panel. Eq. (38) are written at the panel centroids, thus providing a system of $N_{F}^{-}+N_{I}+N_{N}^{-}$equations, with $N_{F}^{-}+2 N_{I}+N_{N}^{-}$unknowns.

A similar system of equations is obtained for the right subdomain, which is

$$
\begin{aligned}
& a_{i} \phi_{i}^{+}+\sum_{j=1}^{N_{N}^{+}} \phi_{j}^{+} d_{i j}+\sum_{j=N_{N}^{+}+1}^{N_{N}^{+}+N_{I}} \phi_{n, j *}^{-} g_{i j}+\sum_{j=N_{N}^{+}+1}^{N_{N}^{-}+N_{I}} \phi_{j}^{+} d_{i j}-\sum_{j=1}^{N_{F}^{+}} \phi_{n, j}^{+} g_{i j} \\
& =e_{i} \phi_{i}^{+}+\sum_{j=1}^{N_{N}^{-}} \phi_{n, j}^{+} g_{i j}-\sum_{j=N_{N}^{+}+N_{I}+1}^{N_{N}^{-}+N_{I}+N_{F}^{+}} \phi_{j}^{-} d_{i j},
\end{aligned}
$$

where $N_{F}^{+}$in this case involves the free surface panels lying on both free surface portions which are the vertical, $x=0, \delta<y<1$, and horizontal, $0<x<20, y=1$ ones. The third contribution in the left hand side of Eq. (39) accounts for condition (34) at the interface. For this reason, instead of $\phi_{n, j}^{+}$, it appears the term $-\phi_{n, j^{*}}^{-}$where $j^{*}$ denotes the same panel as an element of the boundary of the minus subdomain $\partial \Omega^{-}$. With the numbering adopted in the two subdomains, it is $j^{*}=$ $N_{F}^{-}+N_{I}+N_{N}^{+}-j$. Thanks to the use of that condition already in the system, when Eq. (39) is written at the centroids of the panels belonging to the boundary of $\Omega{ }^{+}$, it yields a system of $N_{F}^{+}+N_{I}+N_{N}^{+}$ equations with $N_{F}^{+}+2 N_{I}+N_{N}^{+}$unknowns.

In total, Eqs. (38) and (39) provide a system of $N_{F}^{-}+N_{F}^{+}+$ $2 N_{I}+N_{N}^{-}+N_{N}^{+}$algebraic equations for $N_{F}^{-}+N_{F}^{+}+3 N_{I}+N_{N}^{-}+N_{N}^{+}$ unknowns, as the relation (34) between the normal derivatives at the interface have been already exploited. Additional $N_{I}$ equations are added to the system by enforcing the continuity of the pressure at the interface given by Eq. (35), which gives

$\phi_{i}^{+}-\gamma \phi_{j}^{-}=\gamma\left(y_{j}-\delta\right)-\left(y_{i}-1\right)$, 
where $i$ and $j$, although referring to the same panel, differ as the panels are ordered in different way for the left and right subdomains. As already said, $i$ and $j$ are related by the following relation

$j=N_{F}^{-}+N_{I}+N_{N}^{+}-i$.

The solution of the linear system composed by Eqs. (38)-(40) provides the boundary values of the potentials and their normal derivatives and allows one to derive the velocity potential at any point of the flow domain by using the boundary integral representation. For the purpose of establishing a comparison, the distributions of the velocity potentials along the free surface and at the left and right sides of the interface are used to compute the tangential velocity components which, together with the normal derivatives of the velocity potential, completely describe the velocity field.

A few words are deserved by the discretization adopted for the solution of the problem. The discretization is governed by two parameters: the minimum panel size $a_{m}$ and the growth factor $\epsilon$. There are three regions in the flow domain where the solution is expected to have sharp gradients, and where a careful discretization is needed. The problematic regions are located near the triple point $(0, \delta)$, at the bottom $(0,0)$, and at $(0,1)$. For the boundary portions arriving at those points, the amplitude of the first panel is set equal to $a_{m}$, and the growth factor $\epsilon$ is used to assign the size of the successive panels. As the number of panels is necessarily an integer, a small adjustment of the panel size can be needed. So, if $L$ is the length to be discretized, the number of panel $N$ needed for the discretization is such that

$a_{m}+a_{m} \epsilon+a_{m} \epsilon^{2}+\cdots+a_{m} \epsilon^{N-1}=a_{m} \frac{1-\epsilon^{N}}{1-\epsilon}=L$.

From the above equation we obtain

$\epsilon^{N}=1+\frac{L}{a_{m}}(\epsilon-1)$

and then the number of panels to be adopted is

$N=\frac{\log \left(1+L(\epsilon-1) / a_{m}\right)}{\log \epsilon}$.

In order to account for the truncation, the value of the amplitude $a_{m}$ to be adopted in the discretization is derived as

$a_{m}=L \frac{\epsilon-1}{\epsilon^{N}-1}$.

For the common interface between the two subdomains and for the vertical portion of the free surface, both ends have to be refined. In this case the boundary portion is split into two parts of the same length, and the above procedure is applied to both portions. At the side walls, $x= \pm 20$, ten panels are adopted to discretize the boundaries independently of the height. In the computations, the minimum panel size is $a_{m}=0.00001$ and the growth factor is $\epsilon=1.03$. In Fig. 5, an example of the discretization adopted for the case with $\delta=0.5$ is shown.

\section{Numerical results}

Computations of the velocity field and of the shapes of the two free surface portions and of the interface were performed using both the Fourier series method (FSM) and boundary element method (BEM) for different values of density ratios $\gamma$ and different depth ratios $\delta$. In the computations by BEM, the minimum panel size is $a_{m}=10^{-5}$ and the growth factor is $\epsilon=1.03$. In the computations by FSM, $M=3000$ terms were retained in the system (20) with $N$ equal to the integer part of $6000 / \delta$. It was found that the boundary conditions ( 7 ) on the interface are most difficult to satisfy with good accuracy. Such a big number of retained terms in FSM and such a small size of the panels in BEM were dictated by the interface conditions and required accuracy. Three criteria are used to determine the sufficient number of modes

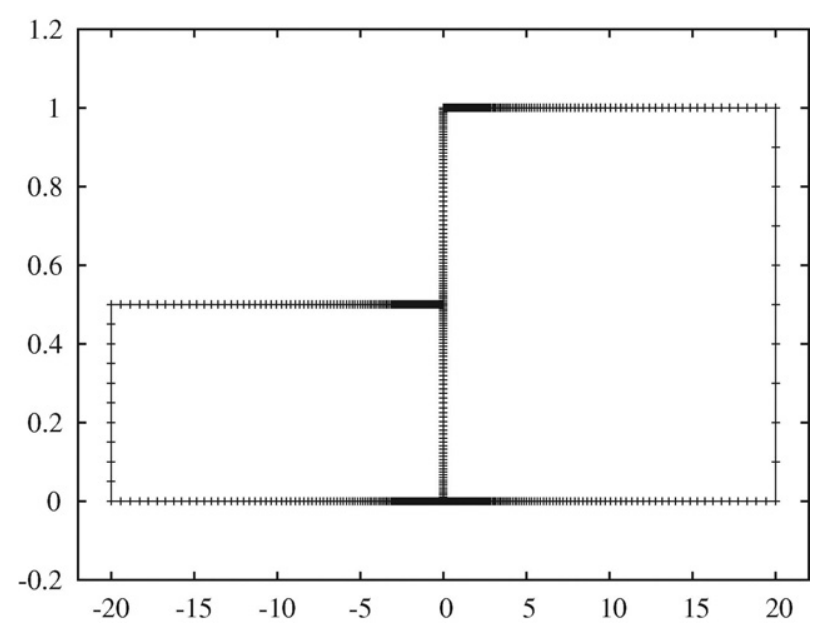

Fig. 5. Discretization adopted for the case $\delta=0.5$. Note that different scales have been adopted for the horizontal and vertical axes.

in FSM: allowed proximity to the triple point along the interface, round off errors and the CPU time. The first criterion implies that the closer we get to the triple point, the more difficult it becomes to satisfy the condition of equality of the horizontal velocities at the interface, second equation in (7). This is expected since the flow near the triple point is power-singular (see Section 4). The second criterion implies that as the number of modes increases, so does the number of equations, which leads to the round off error in the numerical solution. The third criterion implies the increase of CPU time with the increase in number of equations in the system (20). By considering all of this $M=3000$ seems to be the optimum value. With this value of $M$, for $\gamma=1$ and $\delta=0.5$, the relative error in satisfying the boundary conditions ( 7 ) at $y=\delta-0.001$ is $3.3 \%$ and the CPU time is 1206.512 s using a computer with $2.2 \mathrm{GHz}$ CPU. Below the numerical results are presented for both horizontal and vertical velocities of the flows at the interface, the normal velocities of the free boundaries which come to the triple point, and for the coefficient $E_{R}$ of the singularity of the flow velocity at the triple point.

Horizontal velocities at the interface $\phi_{x}^{+}(0, y)$, where $0<y<\delta$, are shown in Fig. 6 for different depth ratios $\delta$.

Eq. (11) provides that the displacement of the interface is proportional to its horizontal velocity at each time instant. The horizontal velocity profiles computed by FSM and BEM are shown for eight values of $\gamma$. It is seen that the agreement between the results by these two methods is very good, except for the case of $\gamma=10$. We know from Section 4 that for very large values of $\gamma$ the behaviour of the fluid on the left is that of dam-break flow at the leading order, which can not be recovered from the algebraic system (20) by setting $\gamma=$ $\infty$. However when $\gamma$ is small, say 0.01 , the dam break flow of the right fluid is well recovered by setting $\gamma=0$ in Eqs. (16) and (17). It is seen that the horizontal velocity of the interface is log-singular at the bottom except when $\gamma=1$, it tends to $-\infty$ as $y \rightarrow 0^{+}$, when $\gamma$ $<1$, and to $+\infty$ when $\gamma>1$. The horizontal velocity tends always to minus infinity when the triple point is approached from below. This singularity disappears when $\gamma \rightarrow 0$. This implies that the initially vertical interface always turns to the left near the triple point, which is expected to be the origin of the jet-like flow started from this point, and resembles the dam-break flow close to the bottom (see Section 4, Eq. (29)). The flow along the bottom for $\gamma=1$ always directed to the left towards the region of smaller depth.

The vertical velocities at the interface $\phi_{y}^{-}(0, y)$ and $\phi_{y}^{+}(0, y)$, where $0<y<\delta$, are shown in Fig. 7 for $\delta=0.5$ and different density ratios $\gamma$. In this figure only the FSM results are shown since the agreement with the BEM results is quite good. 
(a)

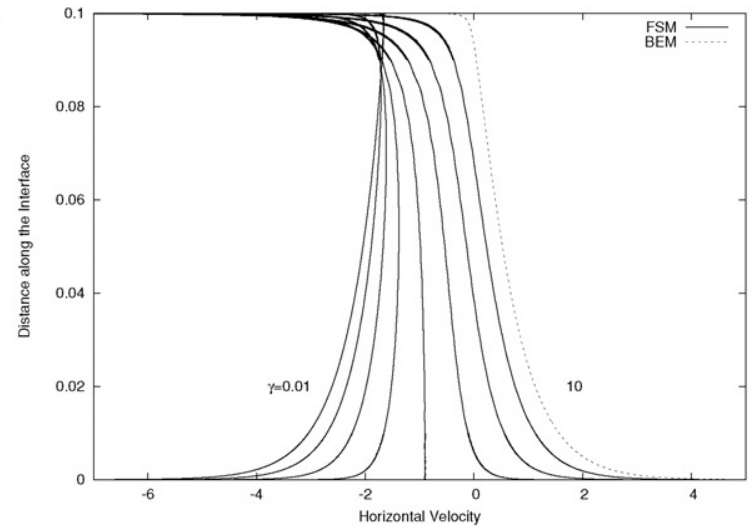

(b)

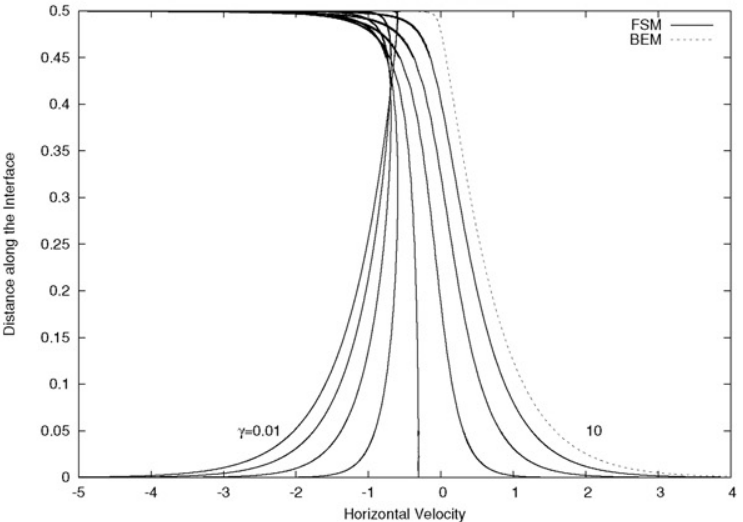

(c)

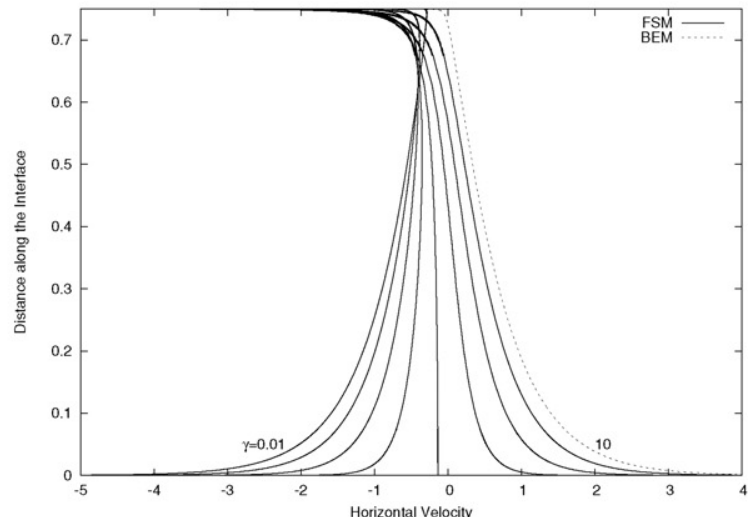

Fig. 6. Horizontal velocities $\phi_{x}^{+}(0, y)$ of the interface as functions of the distance $y$ along the interface from the bottom for $\delta=0.1$ (a), 0.5 (b), and 0.75 (c). In each figure, there are eight curves computed for $\gamma=0.01,0.1,0.25,0.5,1,2,4,10$ by the Fourier series method (solid lines) and boundary element method (dotted lines).

For small $\gamma$ (see Fig. 7a) the vertical velocity of the fluid on the right is approximately -1 , which follows from Eq. (7), and the velocity of the fluid on the left is positive and singular at the triple point as predicted in [15]. Fig. 7b shows that the vertical velocity in the case of two identical fluids is very small everywhere except a vicinity of the corner point $(\delta, 0)$. For a heavier fluid on the left (Fig. 7c), the vertical velocity of this fluid on the interface is negative (compare with Fig. 7a), and the vertical velocity of the lighter fluid on the right is positive. Note that the vertical velocity on the free surface $x=0$, $\delta<y<1$ is negative. This implies that the flows below the triple point and above this point collide at this point with expected jet flow started at this point. Different vertical velocities $\phi_{y}^{-}(0, y)$ and $\phi_{y}^{+}(0, y)$ at the interface indicate high shear stresses and hence instability of the flow near the interface.
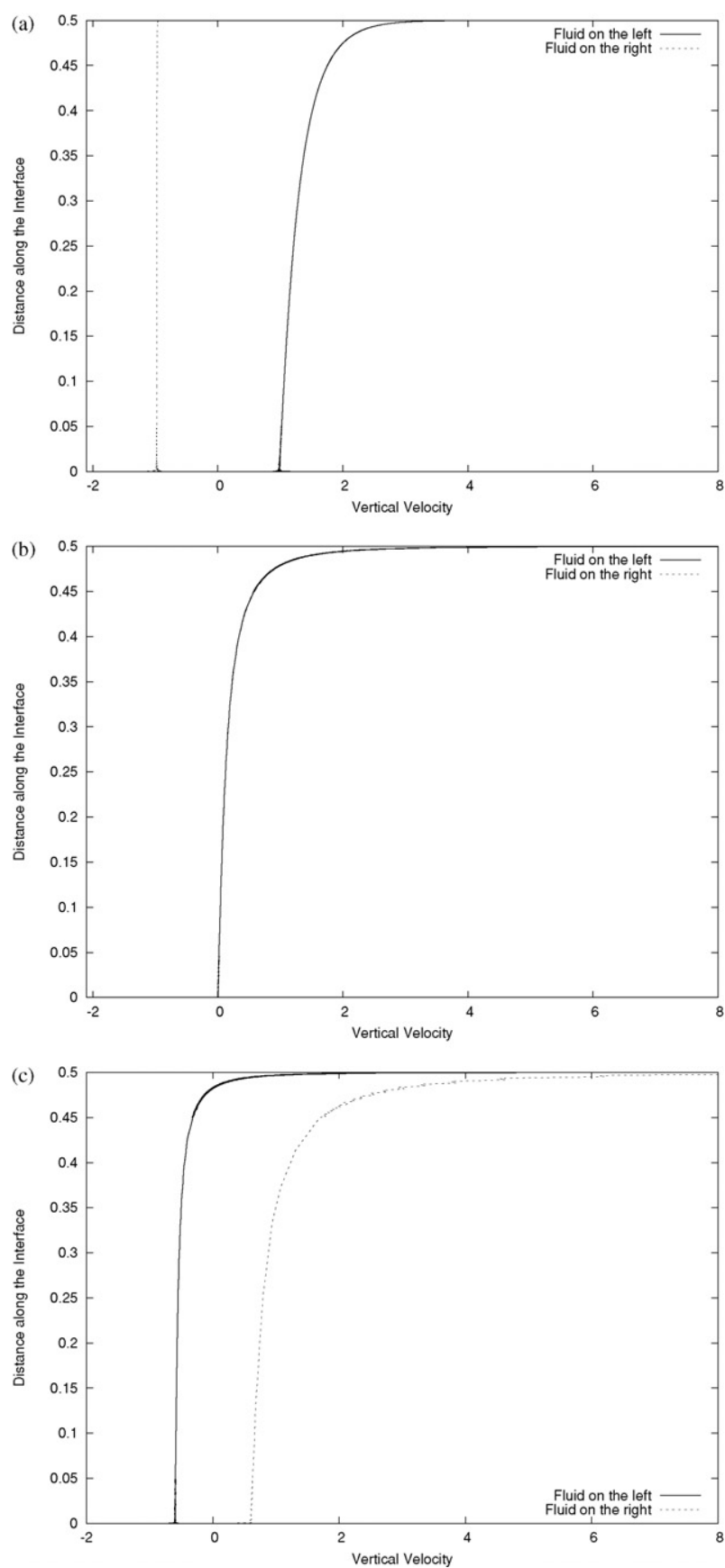

Fig. 7. Vertical velocities $\phi_{y}^{+}(0, y)$ (solid lines) and $\phi_{y}^{-}(0, y)$ (dotted lines) of the flows along the interface as functions of the distance $y$ from the bottom for $\delta=0.5$ and $\gamma=$ 0.01 (a), 1 (b), 4 (c).

Vertical velocities of the initially horizontal free surface $y=\delta, x<$ 0 , are shown in Fig. 8 for $\delta=0.5$ and different density ratios $\gamma$.

It is seen that for $\gamma<1$ the vertical velocity is positive. This implies that the corresponding free surface is deflected upwards where $x<$ 0 and is singular at $x=0$. For small $\gamma$ the motion of the free surface is that studied in [15] but for non-uniform speed of the vertical wall. When $\gamma$ is moderate, $\gamma=1,2$ in the figure, the free surface at a distance from the interface goes down resembling the motion of the free surface in the dam-break flow, but close to the triple point it goes upwards indicating formation of the jet at the triple point. Note that the results by FSM and BEM are different only for $\gamma=10$.

Horizontal velocities of the vertical free surface $x=0, \delta<y<1$ are shown in Fig. 9 for $\delta=0.5$ and different density ratios $\gamma$. 


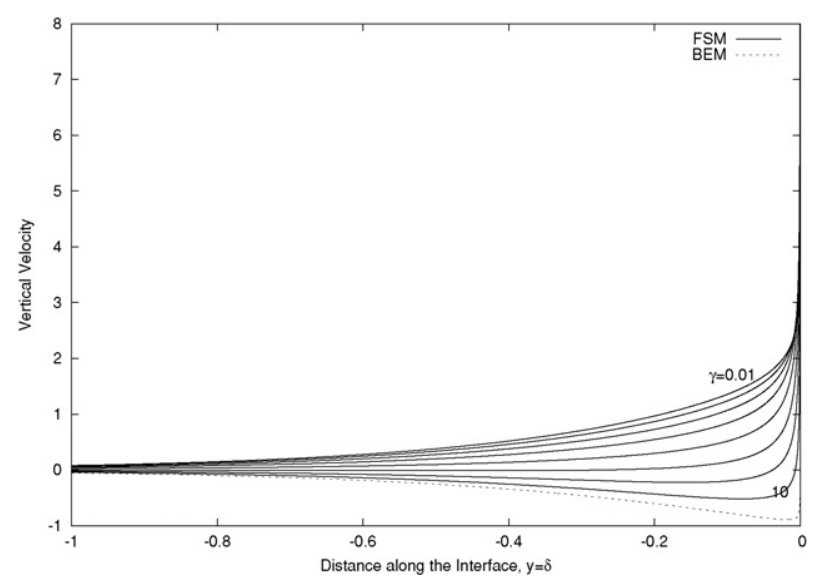

Fig. 8. Vertical velocities $\phi_{y}^{-}(x, \delta)$ of the free surface of the liquid on the left for $\delta=0.5$ and $\gamma=0.01,0.1,0.25,0.5,1,2,4,10$ computed by the Fourier series method (solid lines) and boundary element method (dotted lines).

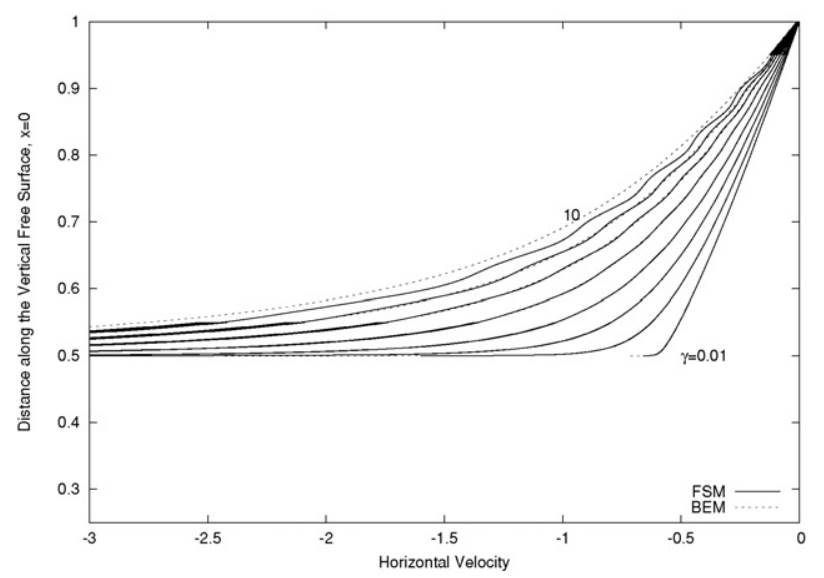

Fig. 9. Horizontal velocities $\phi_{x}^{+}(0, y)$ of the initially vertical free surface of the liquid on the right for $\delta=0.5$ and $\gamma=0.01,0.1,0.25,0.5,1,2,4,10$ computed by the Fourier series method (solid lines) and boundary element method (dotted lines).

It is seen that the horizontal velocity is negative for any $\gamma$. For small $\gamma$ the horizontal velocity is approximately that predicted by the dam-break solution [17] with the liquid on the left being disregarded. With increasing $\gamma$ the horizontal velocity increases as it is seen in the figure. This is due to resistance of the fluid on the left for $\gamma<1$ and due to the flow of this fluid to the right when $\gamma>1$. The horizontal speed is singular at $y=\delta$ for any value of $\gamma>0$. Again, it is worth noticing that the results by FSM and BEM differ only for $\gamma=10$.

The coefficient $E_{R}$ of the singular term in Eq. (30) for the horizontal velocity of the interface is calculated as the limit (33) of $E_{R}^{(n)}$ when $n$ $\rightarrow \infty$. The elements $E_{R}^{(n)}$ are plotted in Fig. 10 as a function of $n$ for $\gamma$ $=1$ and $\delta=0.5$.

The coefficients $d_{n}$ in (33) are obtained as the solutions of the FSM system (20) with $M=3000$ and $N=12,000$. Due to the end effect, the values of $d_{n}$, where $n$ is close to $M$, are not reliable. Fig. 10 shows that $E_{R}^{(n)}$ converges to -0.582 as $n$ approaches 2000 but then diverges due to the end effect. A similar result, -0.5877 , follows from the BEM computations summarised in Table 1 . In this table, horizontal velocities $u^{+}(0, y)$ of the liquid free surface, $x=0, \delta<y<1$, and the interface, $0<y<\delta$, are shown in the second column for different values of the vertical coordinate $y$, in the first column, around the triple point for $\delta=0.5$ and $\gamma=1$. Then Eqs. (25) and (26) were used to compute approximately the coefficient $E_{R}$ in (25).

We can see from Table 1 that the value of $E_{R}$ is around -0.5877 except for the two points which are closest to the triple point $(0, \delta)$.

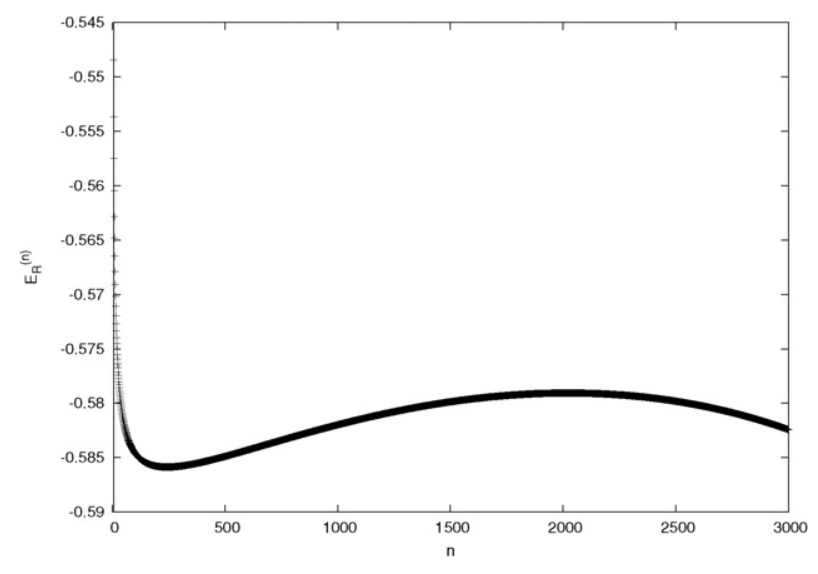

Fig. 10. The coefficient $E_{R}^{(n)}$ obtained from Fourier series analysis. The parameters are $\gamma=1, \delta=0.5$.

Table 1

The coefficient $E_{R}$ in (25) predicted by the boundary element analysis for $\delta=0.5$ and $\gamma$ $=1$.

\begin{tabular}{lcc}
\hline$y$ & $u^{+}$ & $E_{R}$ \\
\hline 0.498836 & -2.785727 & -0.586075 \\
0.499048 & -2.986347 & -0.587556 \\
0.499260 & -3.258201 & -0.589416 \\
0.499472 & -3.667534 & -0.592968 \\
0.499683 & -4.311807 & -0.587874 \\
0.499894 & -7.403372 & -0.699777 \\
0.500109 & -14.658616 & -0.699513 \\
0.500326 & -8.435848 & -0.580677 \\
0.500544 & -7.231771 & -0.590312 \\
0.500762 & -6.434673 & -0.587699 \\
\hline
\end{tabular}

Assuming that the asymptotic representation of velocities near the singular point is correct then we may conclude that the horizontal velocities for the closest two points around the singularity are not reliable in the numerical analysis. Moreover, the value -0.5877 is in very good agreement with the value -0.582 obtained from the Fourier series solution.

To estimate the size $a\left(t^{\prime}\right)$ of the inner region near the triple point, where the linear solution obtained in this paper has to be corrected, we note that the velocity potentials $\varphi^{\prime \pm}\left(x^{\prime}, y^{\prime}, t^{\prime}\right)$ in (1) behave as

$\varphi^{\prime \pm}\left(x^{\prime}, y^{\prime}, t^{\prime}\right) \sim t^{\prime}\left(r^{\prime}\right)^{1-\alpha} \quad\left(r^{\prime} \rightarrow 0\right)$

near the triple point, which follows from the local analysis and Eq. (25). Here $r^{\prime}$ is the distance from the triple point and $a\left(t^{\prime}\right) \rightarrow 0$ as $t^{\prime}$ $\rightarrow 0$. In the inner variables with $r^{\prime}=a\left(t^{\prime}\right) \tilde{r}$ and $\tilde{r}=O(1)$, we find the following orders

$\varphi_{t}^{\prime \pm} \sim a^{1-\alpha}\left(t^{\prime}\right), \quad\left|\nabla \varphi^{\prime \pm}\right|^{2} \sim t^{\prime 2} a^{-2 \alpha}\left(t^{\prime}\right)$

of the two terms in the Bernoulli equation for the hydrodynamic pressure as $t^{\prime} \rightarrow 0$. To allow us to describe the jet formation in the inner region, these two terms must be of the same order. Then

$a\left(t^{\prime}\right)=t^{\prime k}, \quad k(\gamma)=\frac{2}{1+\alpha(\gamma)}$.

Therefore, the inner flow close to the triple point is self-similar with respect to the variable $\tilde{r}=r^{\prime} t^{\prime-k}$ and nonlinear even for small time. The inner flow and the jet formation can be described only numerically. The inner problem is not considered in this paper.

\section{Conclusions}

The linear problem of the initial stage of dam break flow of two immiscible fluids has been solved by the FSM and the BEM. Comparisons between the results of both methods show good agreement 
except for the case when $\gamma$ is very large, say 10 , where the Fourier series method has convergence problems.

It is observed that the horizontal velocity of the interface is logsingular at the bottom except when $\gamma=1$. The horizontal velocity tends always to minus infinity when the triple point is approached from below. This singularity disappears when $\gamma \rightarrow 0$. This implies that the initially vertical interface always turns to the left near the triple point, which is expected to be the origin of the jet-like flow started from this point, and resembles the dam-break flow close to the bottom.

It is discovered that the flow singularity at the triple point is a power singularity, $r^{-\alpha}$ with $\alpha$ being dependent on the density ratio $\gamma$. The higher the value of $\gamma$, the more singular the flow. For $\gamma \neq 1$, the fluids move in different directions at the interface causing shear stress and instability [21]. The jet formation at the triple point is observed by both methods. Fine details of the flow near the triple point are described by the inner solution which is nonlinear and self-similar. The inner problem will be investigated by the methods developed in [13].

\section{Acknowledgement}

This study was carried out with support from TUBITAK (The Scientific and Technological Research Council of Turkey), Grant No. $111 \mathrm{M} 209$.

\section{References}

[1] Stansby PK, Chegini A, Barnes TCD. The initial stages of dam-break flow. Journal of Fluid Mechanics 1998;374:407-24.

[2] Janosi IM, Jan D, Szabo KG, Tel T. Turbulent drag reduction in dam-break flows. Experiments in Fluids 2004;37:219-29.

[3] Gomez-Gesteira M, Rogers BD, Dalrymple RA, Crespo AJC. State-of-the-art of classical SPH for free-surface flows. Journal of Hydraulic Research 2010;48:627.
[4] Lind SJ, Xu R, Stansby PK, Rogers BD. Incompressible smoothed particle hydrodynamics for free-surface flows: a generalised diffusion-based algorithm for stability and validations for impulsive flows and propagating waves. Journal of Computational Physics 2012;231:1499-523.

[5] Özgökmen TM, Iliescu T, Fisher PF, Srinivasan A, Duan J. Large eddy simulation of stratified mixing in two-dimensional dam-break problem in a rectangular enclosed domain. Ocean Modelling 2007;16:106-40.

[6] Goater JN, Hogg AJ. Bounded dam-break flows with tailwaters. Journal of Fluid Mechanics 2011;686:160-86.

[7] Gill AE. Atmosphere-ocean dynamics. California: Academic Press; 1982.

[8] Cooker MJ, Peregrine DH, Vidal C, Dold JW. The interaction between a solitary wave and a submerged circular cylinder. Journal of Fluid Mechanics 1990;215:1-22.

[9] Martinez-Legazpi Aguilo P. Corner waves downstream from a partially submerged vertical plate. PhD thesis, Universidad Carlos III de Madrid; 2011.

[10] Korobkin AA, Wu GX. Impact on a floating circular cylinder. Philosophical Transactions of the Royal Society London 2000;456:2489-514.

[11] Norkin M, Korobkin A. The motion of the free-surface separation point during the initial stage of horizontal impulsive displacement of a floating circular cylinder. Journal of Engineering Mathematics 2011;70(1-3):239-54.

[12] Iafrati A, Korobkin AA. Starting flow generated by the impulsive start of a floating wedge. Journal of Engineering Mathematics 2005;51:99-126.

[13] Iafrati A, Korobkin AA. Initial stage of flat plate impact onto liquid free surface. Physics of Fluids 2004;16(7):2214-27.

[14] Needham DJ, Billingham J, King AC. The initial development of a jet caused by fluid, body and free-surface interaction. Part 2. An impulsively moved plate. Journal of Fluid Mechanics 2007;578:67-84

[15] Peregrine H. Flow due to vertical plate moving in a channel. Unpublished notes; 1972.

[16] Roberts AJ. Transient free-surface flows generated by a moving vertical plate. Quarterly Journal of Mechanics and Applied Mathematics 1987;40:129-58.

[17] Korobkin AA, Yilmaz O. The initial stage of dam-break flow. Journal of Engineering Mathematics 2009;63(2-4):293-308.

[18] Dalrymple RA. Water waves past abrupt channel transitions. Applied Ocean Research 1989;11:170-5.

[19] Hudde H, Letens U. Scattering matrix of a discontinuity with a non rigid wall in a lossless circular duct. Journal of Acoustical Society of America 1985;78:182637.

[20] Gradshteyn IS, Ryzhik IM. Table of integrals, series and products. 7th ed. Amsterdam: Academic Press; 2007.

[21] Hooper AP, Boyd WGC. Shear-flow instability at the interface between two viscous fluid. Journal of Fluid Mechanics 1983;128:507-28. 